\title{
CONSTRAINTS ON X-RAY POLARIZATION OF SYNCHROTRON JETS FROM STELLAR-MASS BLACK HOLES
}

\author{
D. M. RUSSELL \\ Astronomical Institute 'Anton Pannekoek', University of Amsterdam, \\ P.O. Box 94249, 1090 GE Amsterdam, the Netherlands; \\ and \\ Instituto de Astrofísica de Canarias (IAC), E-38205 La Laguna, Tenerife, Spain \\ russell@iac.es
}

\begin{abstract}
For most black hole X-ray binaries, the fraction of X-ray flux originating in the synchrotron jets is generally thought to be low in the hard state. However in one intriguing case, the infrared - X-ray correlations, evolution of broadband spectra and timing signatures suggest that synchrotron emission from a jet likely dominated both the infrared and X-ray flux on the hard state decline of an outburst of XTE J1550-564 at a luminosity of $\sim\left(2 \times 10^{-4}-2 \times 10^{-3}\right) L_{\text {Edd }}$. Synchrotron emission from the relativistic jets launched close to black holes can be highly linearly polarized, depending on the configuration of the magnetic field. It has recently been shown that the polarimetric signature of their jets is detected in the infrared and is highly variable. This reveals the magnetic geometry in a region of the compact jet near its base, close to the black hole. From these results, it is predicted that in some cases, high (possibly up to $10 \%$ ), variable levels of $\mathrm{X}$-ray polarization from synchrotron emission originating in jets will be detected from accreting black holes by future spaceborne X-ray polarimeters.
\end{abstract}

Keywords: accretion; X-rays: binaries; ISM: jets and outflows.

\section{Introduction}

The study of polarized X-rays from astronomical sources is about to be re-born, with upcoming missions such as the Gravity and Extreme Magnetism Small Explorer $(\text { GEMS })^{1}$ and New Hard X-ray Mission (NHXM) ${ }^{2}$. The polarization properties of emission from black hole X-ray binaries (BHXBs) have been well studied at radio frequencies (see Ref. 3 for a review), and have recently been given some attention at optical and infrared (OIR) frequencies. In the optical regime, polarization due to the scattering of intrinsically unpolarized thermal emission can be modulated on the orbital period, which can be used to constrain the physical and geometrical properties of the system ${ }^{4,5}$. At radio, and in some cases at OIR frequencies, variable polarization can be due to synchrotron emission from the jets launched via the process of accretion onto the black hole or neutron star $6,7,8,9$.

More than thirty years ago, a few measurements of polarization from X-ray binaries were made at X-ray energies using the Bragg crystal polarimeters aboard the OSO 8 satellite. For example, the linear polarization of Cygnus X-1 was measured 
to be $2-5 \%$ at $2.5-5.2 \mathrm{keV}^{10}$. Since then, no X-ray detector has had the capabilities to measure the polarization properties of X-ray binaries more accurately. Only recently has the INTEGRAL satellite been used, using a novel technique, to estimate the polarization of the $\mathrm{Crab}^{11}$ and Cygnus $\mathrm{X}-1$ at $\gamma$-ray energies ${ }^{12}$. However in the coming years, promise of new X-ray polarization capabilities are coming in the form of GEMS and NHXM, whose polarimeters will be able to measure linear polarization at energies of $2-10 \mathrm{keV}$ and $2-35 \mathrm{keV}$ respectively, to higher levels of accuracy than achieved before ${ }^{1,2}$.

Here, the X-ray polarization one may expect to detect from synchrotron emission from the jets of X-ray binaries is investigated. Two recent, appropriate results are:

- Sometimes, synchrotron emission from jets can contribute to, and occasionally dominate, the X-ray flux of a black hole X-ray binary,

- This synchrotron emission is polarized at a low, and variable level.

From the combination of these results, it is shown here that future spaceborne X-ray polarimeters like GEMS and NHXM are likely to detect variable X-ray polarization from synchrotron emission from the jets released from accreting Galactic black holes. The polarization properties can help us understand jet physics - in particular, the ordering, orientation and stability of the magnetic field in a region of the jet near its base. The results will have direct implications towards understanding how accretion in the strong gravitational fields close to black holes can result in the launching of relativistic jets.

\section{Evidence for a jet contribution to the X-ray flux}

The origin of the X-ray emission in X-ray binaries is still debated. When the Xray spectrum is well described by a soft, thermal blackbody, the origin is usually attributed to the inner, hot regions of the accretion disc (one exception is the neutron star surface $)^{13}$. In the case of BHXBs, this soft thermal component often dominates (the X-ray 'soft state') and when it does not, the X-ray spectrum can usually be described by a hard power law (the X-ray 'hard state'; e.g. Ref, 14). The inner, hot, possibly radiatively inefficient accretion flow/'corona' is generally considered to produce this power law, due to Compton upscattering of soft photons on hot electrons ${ }^{13}$.

It was first proposed by Ref. 15 that optically thin synchrotron emission from the jet dominates the X-ray flux of the BHXB XTE J1118+480. This was based on broadband, radio to X-ray spectral modelling, where the hard power law at X-ray energies could instead be explained by optically thin jet emission extending from the optical regime. These and similar models were developed to explain the broadband spectral energy distributions (SEDs) of BHXBs in the hard state, and it was shown that the synchrotron component probably produces a significant fraction of the X-ray flux in the hard state, but may not dominate ${ }^{16,17,18,19}$. 
More recently, empirical evidence for the jet producing the hard X-ray power law has been found in the BHXB XTE J1550-564. Here, the jet emission at OIR frequencies was isolated from the emission from the accretion disc and companion $\operatorname{star}^{20}$. This jet component was found to have a spectral index consistent with optically thin synchrotron emission during the fading hard state of its outburst in 2000. The near-infrared (NIR) emission from the jet was also found to be linearly proportional to the simultaneous X-ray flux ${ }^{20}$ (see Fig. 1, upper panel). Moreover, the spectral index between X-ray and OIR, the spectral index of the jet component
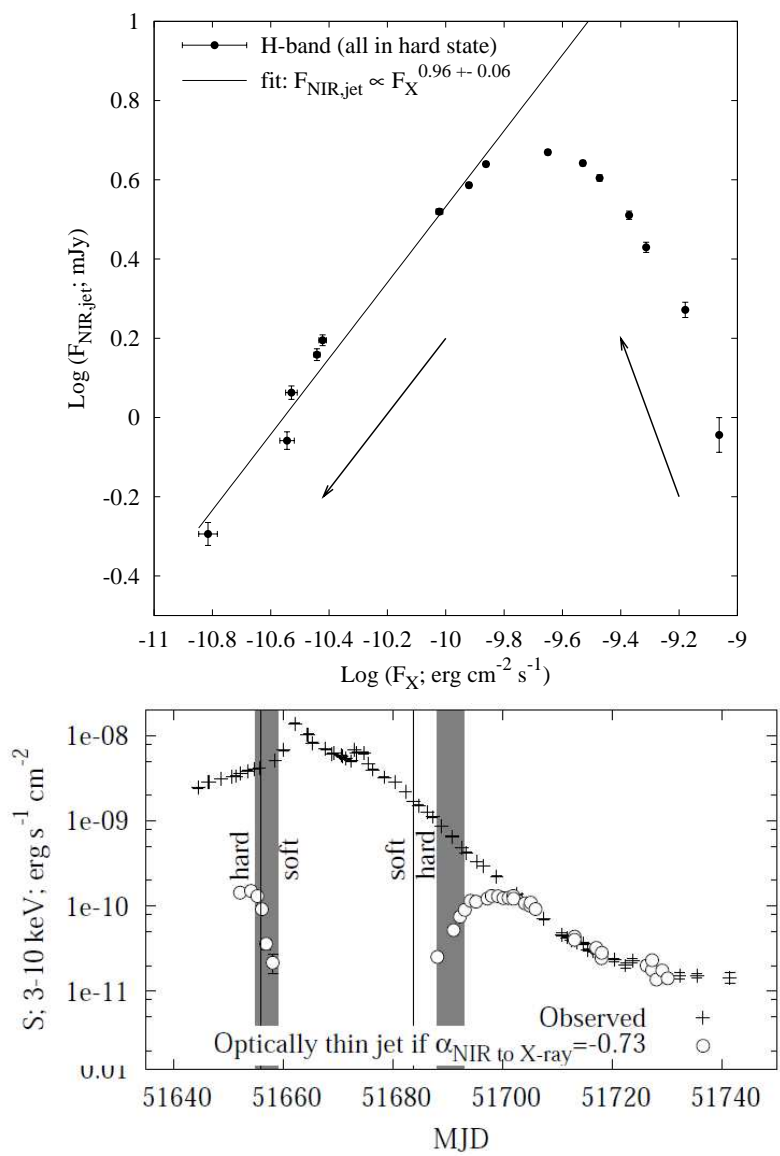

Fig. 1. Upper panel: The NIR-X-ray correlation of XTE J1550-564, using data from the decay of its outburst in 2000. The NIR H-band emission from the jet only is used (the accretion disc and companion star emission are subtracted). The NIR jet emission and the X-ray flux are directly proportional, once both are fading together in the hard state. Before this time, it takes several days for the jet to recover and rise in flux, while the X-ray flux is fading in the hard state. Lower panel: X-ray light curve and possible contribution from the optically thin jet, extrapolated from the OIR regime. Grey regions indicate times in which the jet was fading or recovering at OIR frequencies. Figures from Russell et al. (2010). 
measured at OIR frequencies and the X-ray spectral index itself, were all consistent with the same value. This implies that the broadband spectrum from OIR to X-ray energies is consistent with one single power law fading by one order of magnitude in flux (Fig. 1, lower panel).

The results suggest that the OIR-X-ray power law originated in the optically thin part of the jet synchrotron spectrum during the fading hard state. Initially in the fading hard state the jet flux took several days to brighten, before fading. At a luminosity of $\sim\left(2 \times 10^{-4}-2 \times 10^{-3}\right) L_{\text {Edd }}$, the OIR and X-ray fluxes faded together and were linearly proportional. Before this time, while the jet was brightening, the X-ray power law must have originated in a different component - most likely Compton upscattering from the corona. Evidence to support this change in the source of the dominating X-ray emission comes from an X-ray excess in the light curve over an exponential decay, at the point in which the jet would start to dominate, a slight change in the X-ray hardness, and an increase in the X-ray rms variability.

A similar, but subtly different result has come from multiwavelength monitoring of the BHXB XTE J1752-223 ${ }^{21}$. Here, the jet and accretion disc emission at OIR frequencies were isolated during the decay of its 2009-2010 outburst using the same method as Ref. 20. The optical jet emission was found to rise and fade during the fading hard state, in a similar fashion to XTE J1550-564. In the case of XTE J1752-223, the jet was contemporaneous with a clear X-ray flare with the same morphology in the light curve, implying again a common emission mechanism. The OIR-X-ray spectral index was consistent with optically thin synchrotron emission, but the X-ray timing and spectral properties before and during the flare were the same within errors. This implies that either the jet and corona have very similar emitting properties, or that the X-ray emission was not dominated by the jet (the X-ray emitting component must have been well correlated with the jet though) ${ }^{21}$.

Some additional works have also shown evidence for two components producing the X-ray power law in the hard state, one of which could be the jet. This was demonstrated for the plateau state of GRS 1915+105 (which is equivalent to the canonical hard state) $)^{22,23}$ and for GX 339-4 and GRO J1655-40 at low luminosities in the hard state ${ }^{24}$. In H1743-322, a change in the X-ray emission mechanism was also implied by the emission becoming radiatively efficient above a critical X-ray luminosity $^{25}$. Finally, two power laws were fitted to the X-ray to $\gamma$-ray spectrum of Cyg $\mathrm{X}-1$, one which was about one order of magnitude fainter than the other at $\mathrm{X}$-ray energies ${ }^{12}$.

The above evidence suggests that at some stages of a BHXB outburst, the majority of the X-ray flux originates in optically thin synchrotron emission from the compact jets in the system.

\section{Polarization of optically thin synchrotron emission from the jet}

Optically thin synchrotron emission is intrinsically polarized. If the local magnetic field in the emitting region is ordered, a net linear polarization is observed. If the field 
is tangled, the differing angles of the polarized light dampen the observed, average polarization. The maximum polarization strength is $70 \%$, in the case of a perfectly ordered field ${ }^{26}$. When the radio emission of BHXBs is consistent with optically thin synchrotron, this polarization signature is commonly detected at levels up to tens of per cent ${ }^{6,7}$. This typically happens over soft-to-hard state transitions, and the emission here is from discrete jet ejections.

The continuous, compact jets produce optically thick (self-absorbed) synchrotron emission at radio frequencies. Optically thin synchrotron emission from the compact jet can only be observed at frequencies higher than the 'jet break', between the two spectral regimes, which is usually considered to occur in the IR (e.g. Ref. 15) and has been observed directly in a few sources ${ }^{27,28}$.

In the last few years, observations of this polarized synchrotron emission from the compact jet have been made for the first time. Intrinsic NIR linear polarization was detected from two BHXBs (GRO J1655-40 and GX 339-4) and two neutron star XBs (Sco X-1 and Cyg X-2) ${ }^{8,9,29}$. The polarization was not consistent with an interstellar origin, nor was it due to scattering within the X-ray binary, because the polarization was stronger in the redder filters. For the above two alternative origins one would expect the polarization strength to increase with frequency. Moreover, the polarization of Sco X-1 and GX 339-4 was found to be variable on short timescales, and sometimes consistent with being absent. For GX 339-4, when the polarization was strong the polarization angle was perpendicular to the known jet axis, implying a magnetic field that is parallel to the jet axis. The low levels of polarization measured $(\sim 1-7 \%)$ and variability imply a tangled, rapidly changing magnetic field geometry near the base of the jet ${ }^{8,9}$. This is consistent with recent work ${ }^{28}$ that showed from mid-IR variability of GX 339-4 that either the acceleration region near the base of the jet, or its magnetic field strength, are changing on timescales of minutes (the same timescale as the polarization variability). Finally, we note that Ref. 12 claim a $\gamma$-ray polarization in $\mathrm{Cyg} \mathrm{X}-1$ averaged over several years of $67 \pm 30 \%$, with a possible jet origin. This would imply a very ordered, and stable magnetic field, mis-aligned with the jet axis.

\section{Conclusions}

It has been demonstrated that synchrotron radiation from the jets of BHXBs can sometimes produce the observed X-ray power law in X-ray binaries. From optical and NIR observations, it has been shown that this synchrotron emission is polarized at a low, but variable level (up to $\sim 10 \%$ ). The same distribution of electrons may produce the OIR to X-ray power law, so one could expect this variably polarized emission to be detected by upcoming X-ray polarimeters. In particular, GEMS and NHXM are expected to possess the required sensitivity for Galactic BH transients.

\section{Acknowledgments}

This research was partly supported by a Netherlands Organisation for Scientific Research (NWO) Veni Fellowship and partly by a Marie Curie Intra European 
Fellowship within the 7th European Community Framework Programme under contract no. IEF 274805.

\section{References}

1. J. K. Black et al., SPIE 7732, 25 (2010).

2. G. Tagliaferri et al., Experimental Astron., in press (2011).

3. R. P. Fender, in Compact Stellar X-Ray Sources $\mathbf{3 8 1}$ eds. W. H. G. Lewin and M. van der Klis, (Cambridge University Press, Cambridge, 2006).

4. J. F Dolan and S. Tapia, Publ. Astron. Soc. Pacific 101, 1135 (1989).

5. M. Gliozzi et al., Astron. Astrophys. 337, L39 (1998).

6. D. C. Hannikainen et al., Astrophys. J. 540, 521 (2000).

7. C. Brocksopp et al., Mon. Not. R. Astron. Soc. 378, 1111 (2007).

8. D. M. Russell and R. P. Fender, Mon. Not. R. Astron. Soc. 387, 713 (2008).

9. D. M. Russell et al., in Proceedings of 'High Time Resolution Astrophysics IV - The Era of Extremely Large Telescopes - HTRA-IV', Proceedings of Science. ed. A. Nikolaos (Crete, Greece, 2011a) [arXiv:1104.0837].

10. K. S. Long, G. A. Chanan and R. Novick, Astrophys. J. 238, 710 (1980).

11. A. J. Dean et al., Science 321, 1183 (2008).

12. P. Laurent et al., Science 332, 438 (2011).

13. M. Gilfanov, 2009, in The Jet Paradigm - From Microquasars to Quasars 794, ed. T. Belloni, Lect. Notes Phys. (2010).

14. T. M. Belloni, in The Jet Paradigm - From Microquasars to Quasars 794, ed. T. Belloni, Lect. Notes Phys. (2010).

15. S. Markoff, H. Falcke and R. Fender, Astron. Astrophys. 372, L25 (2001).

16. S. Markoff et al., Astron. Astrophys. 397, 645 (2003).

17. S. Markoff, M. A. Nowak and J. Wilms, Astrophys. J. 635, 1203 (2005).

18. S. Migliari et al., Astrophys. J. 670, 610 (2007).

19. D. Maitra et al., Mon. Not. R. Astron. Soc. 398, 1638 (2009).

20. D. M. Russell et al., Mon. Not. R. Astron. Soc. 405, 1759 (2010).

21. D. M. Russell et al., Mon. Not. R. Astron. Soc., in press [arXiv:1109.3654] (2011b).

22. J. Rodriguez et al., Astrophys. J. 675, 1436 (2008a).

23. J. Rodriguez et al., Astrophys. J. 675, 1449 (2008b).

24. M. A. Sobolewska et al., Mon. Not. R. Astron. Soc. 417, 280.

25. M. Coriat et al., Mon. Not. R. Astron. Soc. 414, 677 (2009).

26. C.-I. Björnsson and G. R. Blumenthal, Astrophys. J. 259, 805 (1982).

27. S. Migliari et al., Astrophys. J. 710, 117 (2010).

28. P. Gandhi et al., Astrophys. J. Lett. 740, L13 (2011).

29. T. Shahbaz et al., Astrophys. J. 672, 510 (2008). 